\title{
Precision of Vegetation Height Estimation using the Dual-Baseline PolInSAR System and RVoG Model with Temporal Decorrelation
}

\author{
Hélène Sportouche, Antoine Roueff and Pascale C. Dubois-Fernandez
}

\begin{abstract}
Estimating vegetation height from PolInSAR data using the RVoG model has motivated several studies. Most of these propose estimators and apply them to real data to demonstrate their potential. In previous publications on the single-baseline system, we proposed a complementary approach which consisted in analyzing the precision of estimations of vegetation height that can be expected depending on the considered model and on the available a priori knowledge. In this paper, we develop such an analysis for the case of a dual-baseline system.

We consider the dual-baseline configuration with a PolInSAR set obtained with three PolSAR acquisitions, the extinction coefficient of the volume is assumed unknown and the level of temporal decorrelation is assumed to be unknown. The observed high sensitivity of the vegetation height Cramer-Rao bound (CRB) with respect to the system parameters and the vegetation characteristics shows that the the system optimization cannot guarantee one meter precision for all vegetation heights, even for large estimation windows with $N=2000$ pixels. Nevertheless, an operating regime exists for which the vegetation height estimation precision is around $1 \mathrm{~m}$ for $N=200$ pixels. This regime is obtained for a pair of wavenumbers $\left(0.06 \mathrm{~m}^{-1}\right.$ and $\left.0.25 \mathrm{~m}^{-1}\right)$, for vegetation height ranging $[20,50] \mathrm{m}$, and for polarimetric contrast between the ground and the volume larger than 0.3. Furthermore, we investigate the performance of a Maximum-Likelihood estimator and compare this to the precision given by the CRB. For the examples considered, with $N=200$ pixels, we observed convergence issues of the estimator when the polarimetric contrast is smaller or equal to 0.3 .
\end{abstract}

\section{INDEX TERMS}

Polarimetric SAR Interferometry (PolInSAR), dualbaseline, Random Volume over Ground (RVoG) model, temporal decorrelation, vegetation height estimation, Cramer-Rao bound (CRB).

During this work, Hélène Sportouche was with Aix Marseille Univ, CNRS, Centrale Marseille, Institut Fresnel, Marseille, France, as a postdoctoral researcher with a CNES (Centre National d'Études Spatiales) grant.

A. Roueff is with Aix Marseille Univ, CNRS, Centrale Marseille, Institut Fresnel, Marseille, France.

P. C. Dubois-Fernandez is with the Department of Electromagnetism and Radar, Office National d'Études et de Recherches Aerospatiales, 13661 Salon-de-Provence, France.

\section{INTRODUCTION}

Worldwide biomass monitoring is essential for a better understanding of the carbon cycle and can be performed from synthetic aperture radar (SAR) measurements [1]. Because the radar backscattered signal saturates for high biomass depending on the radar frequency, a direct biomass estimation from intensity data is not always possible. An alternative approach consists in first estimating forest height using polarimetric interferometric SAR (PolInSAR) techniques [2] and then estimating the biomass through allometric relationships [1]. Being able to correctly retrieve the vegetation height is thus of great importance for biomass estimation.

Different methods have been proposed in the literature to estimate vegetation height from PolInSAR data, depending on the considered configuration and on the available a priori knowledge. In the single-baseline (SB) PolInSAR configuration, corresponding to the acquisition of two PolSAR measurements, vegetation height estimators have been successfully applied on real data at different bands such as L and P [3], [4], [5], [6], [7]. In most cases, these estimators are based on the Random Volume Over Ground (RVoG) model, introduced by Treuhaft et al [8]. To build an estimator, Tabb et al proposed to use a maximumlikelihood based approach [9], [10], which allows one to estimate not only vegetation and ground heights but also polarimetric coherency matrices of the ground and of the volume. Another estimation approach proposed in [3] by Papathanassiou et al provides in addition a geometric interpretation of the complex coherence observed for different polarization states. Afterwards, several variants of the estimator from [3] have been developed, depending on the considered model and on the available a priori knowledge. Initially in [3], [4], the authors proposed to estimate vegetation height and extinction coefficient, under the assumption that, at L-band, the effective ground-tovolume ratio is equal to zero for one polarimetric channel in order to solve the ambiguity problem encountered for this kind of SB approaches with such a model [11], [12]. Alternatively, at P-band, when the ground has a significant contribution for all polarizations, SB height estimation is performed in [6], [13], assuming that the extinction coefficient is known a priori.

Furthermore, it has been shown in [12], [14] that the temporal decorrelation phenomenon, which often occurs in the repeat-pass scenario, can have a significant influence 
on the height retrieval and thus should be taken into account. In [5], the RVoG model with volume temporal decorrelation (called RVoG+VTD) has been introduced and allows the estimation of the vegetation height and of a temporal decorrelation coefficient in SB, assuming not only a negligible ground-to-volume ratio, but also an a priori known extinction coefficient [15]. More recently, another alternative to address temporal decorrelation has been proposed based on a Random Motion over Ground (RMoG) model [16], [7]. In these articles, Lavalle et al, generalizing the works of Zebker and Villasenor [17], consider unstable scatterers located at the ground layer as well as in the volume layer, with a motion variance changing along the vertical axis. In [7], the authors use an objective function with five observations of the temporal and volumetric complex coherence, to inverse in SB the vegetation height, the extinction coefficient and the motion standard deviations of ground and canopy elements from real airborne SAR data at L-band. In [18], Lei et al combine the RMoG model with the effects of dielectric changes for large temporal baselines and provide a simplified height inversion procedure from SB HV-polarized InSAR correlation magnitude data, giving an alternative approach when full-polarization data are not available.

To complement these single-baseline approaches, dualbaseline (DB) configurations have also been considered [19], [20], [21], [22], [23], [24]. In [19] and [20], the introduction of a second spatial baseline allows the development of two DB methods under the RVoG model (respectively a geometrical one and a formal one with norm minimization) using at least four complex coherence observations (i.e. considering at least two polarization channels per baseline) for vegetation height and extinction coefficient estimation, without the zero ground-to-volume ratio hypothesis applied in SB [4]. Moreover, in [22] and in [23], two DB inversion approaches (respectively a geometrical one and a formal one with vectorial criterion minimization), developed under the RVoG+VTD model and also based on complex coherence observations, enable the simultaneous estimation of vegetation height, extinction coefficient and two volume temporal decorrelation factors, but this time conserving a zero ground-to-volume ratio hypothesis. In [24], a DB inversion approach, developed under the RMoG model and using six complex coherence observations provided by the three available spatial baselines, enables to simultaneously estimate vegetation height, extinction coefficient and six temporal decorrelation parameters

Finally, multi-baseline (MB) approaches with larger number of acquisitions have also been considered. In [25], several forest characteristics (including in particular a temporal decorrelation parameter for each baseline) are retrieved from MB PolInSAR data by a joint exploitation of polarimetric and interferometric diversities. In [26], two temporal decorrelation coefficients relative to the ground and volume layers are quantified and their impacts on MB PolInSAR vegetation height estimation are studied. Moreover, in [27] and [28], MB InSAR coherence and phase observations are used for the retrieval of vertical vegetation density profiles. Eventually, MB InSAR or PolInSAR data have been also widely analyzed to develop tomographic techniques [29], [30], [31], [32], [33], [34], requiring usually at least six PolSAR measurements.

Most of these analysis focus on proposing estimation algorithms which are applied to real data to demonstrate their potential, using in situ measurements or LIDAR measurements as ground truth. In this paper, as in the recent studies in [35], [36], [37], we propose a complementary approach which consists in analyzing the precision of vegetation height estimation depending on the considered model and on the available a priori knowledge. More precisely, we explore the amount of information present in the data based on the Cramer-Rao bound (CRB) as a function of the system configuration and as a function of some vegetation characteristics. In particular, we analyse the sensitivity of the CRB with respect to the vertical wavenumbers, the vegetation height, the extinction coefficient, the volume coherence and the polarization properties of the volume and of the ground. The CRB is a bound on the variance of unbiased estimators [38] for a given model and a priori knowledge. It is often used as a reference for precision. It does not depend on the choice of the considered estimator. In [35], it has been shown that the $\mathrm{CRB}$ of the vegetation height can be very high in SB, and thus that the corresponding precision on the vegetation height can be very poor, but in [36] it has also been shown that some a priori knowledge can allow the precision to be improved significantly. More recently in [37], it has been shown that the CRB of the vegetation height depends only on four unknown parameters, which allows one to identify the contrast parameters which influence the precision of the considered estimation problem.

In this paper, we apply this methodology based on the $\mathrm{CRB}$ to the dual-baseline configuration to explore whether adding a third PolSAR image allows one to estimate accurately the vegetation height when extinction coefficient and temporal coherence are unknown. Furthermore, we investigate whether a maximum-likelihood (ML) estimator can reach the precision given by the CRB. Thus, we consider a DB PolInSAR model, which takes into account temporal decorrelation of the volume, based on the RVoG+VTD model [5], with unconstrained polarimetric coherency matrices.

We show via simulations that, the precision described by the vegetation height CRB is sensitive not only to system configuration (vertical wavenumbers), but also to the vegetation characteristics (in particular vegetation height and polarimetric properties). Thus, optimizing the system to obtain a one meter precision on vegetation height estimation for all vegetations seems impossible. However, we show an operating regime exists for which the vegetation height estimation precision is around $1 \mathrm{~m}$ for $N=200$ pixels and for vegetation heights between 20 and $50 \mathrm{~m}$, which corresponds to a large percentage of the higher biomass forest. Section II details the model, the CRB computation and the implemented maximum likelihood 
(ML) estimator. Then, the evolutions of the vegetation height $\mathrm{CRB}$ as a function of the system configuration and of the vegetation characteristics are analyzed in section III. Finally, in section IV, the main conclusions of the paper are drawn and some perspectives are considered.

\section{CRAMER-RAO BOUND AND}

MAXIMUM-LIKELIHOOD BASED ESTIMATOR WITH THE DUAL-BASELINE CONFIGURATION AND RVOG MODEL

\section{A. Dual-baseline RVoG model}

For each pixel of a DB PolInSAR image, a 9D complex valued vector $\boldsymbol{k}$ is defined as the concatenation of three PolSAR measurements $\boldsymbol{u}_{i}$ by $\boldsymbol{k}=\left[\boldsymbol{u}_{1}^{T}, \boldsymbol{u}_{2}^{T}, \boldsymbol{u}_{3}^{T}\right]^{T}$, where $\boldsymbol{u}_{i}$ can be written in the Pauli basis as $\boldsymbol{u}_{i}=\frac{1}{\sqrt{2}}\left[S_{i}^{H H}+\right.$ $\left.S_{i}^{V V}, S_{i}^{H H}-S_{i}^{V V}, 2 S_{i}^{H V}\right]^{T}$ and where $S_{i}^{Y X}$ denotes the complex scattering amplitude measured at antenna $i$ for a given $X$ emitting polarization state and a given $Y$ receiving polarization state. Introducing $\boldsymbol{T}_{i j}=\left\langle\boldsymbol{u}_{i} \boldsymbol{u}_{j}^{\dagger}\right\rangle$, where $\langle$.$\rangle is the statistical expectation operator, the 9 \times 9$ covariance matrix of $\boldsymbol{k}$ has the following form:

$$
\boldsymbol{\Upsilon}=\left\langle\boldsymbol{k}(\boldsymbol{k})^{\dagger}\right\rangle=\left(\begin{array}{lll}
\boldsymbol{T}_{11} & \boldsymbol{T}_{12} & \boldsymbol{T}_{13} \\
\boldsymbol{T}_{12}^{\dagger} & \boldsymbol{T}_{22} & \boldsymbol{T}_{23} \\
\boldsymbol{T}_{13}^{\dagger} & \boldsymbol{T}_{23}^{\dagger} & \boldsymbol{T}_{33}
\end{array}\right) .
$$

where $\left(\boldsymbol{T}_{i j}\right)_{(i, j) \in\{1,2,3\}^{2}}$ are $3 \times 3$ matrices.

We assume that the vegetation can be described by a homogeneous Random Volume over Ground (RVoG) model [8] with temporal decorrelation in the volume layer similarly to [5]. Thus, introducing $\rho_{i j}$ as the temporal correlation coefficient (equivalently $\left(1-\rho_{i j}\right)$ is called the temporal decorrelation coefficient [5]) between antenna $i$ and antenna $j$ (where $\rho_{i i}=1 \quad \forall i \in\{1,2,3\}$ ), $k_{i j}^{z}$ as the vertical sensitivity (or vertical wavenumber) between antenna $i$ and antenna $j$ (where $k_{i i}^{z}=0 \quad \forall i \in\{1,2,3\}$ ), and $\boldsymbol{T}_{\text {vol }}$ and $\boldsymbol{T}_{\text {gro }}$ as respectively the polarimetric coherency matrices associated with volume and ground, for $(i, j) \in\{1,2,3\}^{2}$, the matrix $\boldsymbol{T}_{i j}$ can be written:

$$
\boldsymbol{T}_{i j}=e^{\mathrm{j} k_{i j}^{z} z_{i j}}\left(\rho_{i j} I_{i j} \boldsymbol{T}_{v o l}+a \boldsymbol{T}_{g r o}\right) .
$$

Note that with this model, the coherency matrices $\boldsymbol{T}_{v o l}$ and $\boldsymbol{T}_{\text {gro }}$ do not depend on the baseline $i j$. Moreover, in Eq. (2), $I_{i j}$ and $a$ are defined by:

$$
I_{i j}=\frac{e^{\mathrm{j} k_{i j}^{z} h_{v}}-e^{-\alpha h_{v}}}{\mathrm{j} k_{i j}^{z}+\alpha}, a=e^{-\alpha h_{v}} \text { with } \alpha=2 \sigma_{v} / \cos \beta,
$$

where $\sigma_{v}$ is the polarization-independent and vertically constant extinction coefficient of the volume, $h_{v}$ is the vegetation height and $\beta$ is the incident angle of the radar [4]. Note that in Eq. (2), the ground height parameter $z_{i j}$ depends on the indices $(i, j)$. Obviously, the true ground height does not depend on the interferometric indices $(i, j)$. However, due to phase calibration issues, the interferometric phase associated with the indices $(i, j)$ may introduce an error in the measured ground height [39], [40]. As a result, knowledge on the SB ground phase cannot be used as input information for another pair as it can be affected by this phase bias. To take into account this uncertainty, we introduce a ground height $z_{i j}$ that depends on the interferometric pair.

In the considered model, the system parameters $\beta$ and $\left(k_{i j}^{z}\right)_{\{(i, j)=(1,2),(2,3),(1,3)\}}$ are known from the experimental setup, while the other parameters are unknown and have to be estimated in an homogeneous region where measurements are identically distributed. Moreover, introducing the interferometric ground phase $\phi_{i j}=k_{i j}^{z} z_{i j}$, it can be shown [41] that $k_{13}^{z}=k_{12}^{z}+k_{23}^{z}$ and $\phi_{13}=\phi_{12}+\phi_{23}$, thus

$$
z_{13}=\frac{1}{k_{13}^{z}}\left(k_{12}^{z} z_{12}+k_{23}^{z} z_{23}\right),
$$

which means that the number of unknown ground heights (or similarly ground phases) can be reduced to two.

In this paper, we generate data for which numerical values of parameters verify the particular case:

$$
z_{12}=z_{23} \text { and } \rho_{i j}=\rho \text { for } i \neq j .
$$

We make this assumption to be able to compare the estimation precisions as a function of different a priori knowledges: situations for which estimations are obtained using the knowledge of both equations in Eq. (5), but also when it is not the case. In particular, when the estimator does not use this a priori knowledge, it may estimate three temporal coherences and two ground heights. The four considered situations correspond to different vectors of unknown parameters:

$$
\begin{aligned}
& \boldsymbol{\theta}_{22}=\left\{\left(t_{v o l, k}\right)_{k=1, \ldots, 9}, \quad\left(t_{g r o, k}\right)_{k=1, . ., 9}, z_{12}, h_{v}, \sigma_{v}, \rho\right\} \\
& \quad \text { with } z_{12}=z_{23} \text { and } \rho_{i j}=\rho \text { for } i \neq j, \\
& \boldsymbol{\theta}_{23}=\left\{\left(t_{v o l, k}\right)_{k=1, . ., 9}, \quad\left(t_{g r o, k}\right)_{k=1, . ., 9}, z_{12}, z_{23}, h_{v}, \sigma_{v}, \rho\right\} \\
& \quad \text { with } \rho_{i j}=\rho \text { for } i \neq j, \\
& \boldsymbol{\theta}_{24}=\left\{\left(t_{v o l, k}\right)_{k=1, . ., 9}, \quad\left(t_{g r o, k}\right)_{k=1, . ., 9}, z_{12}, h_{v}, \sigma_{v},\right. \\
& \left.\quad \rho_{12}, \rho_{23}, \rho_{13}\right\} \quad \text { with } z_{12}=z_{23}, \\
& \boldsymbol{\theta}_{25}=\left\{\left(t_{v o l, k}\right)_{k=1, . ., 9}, \quad\left(t_{g r o, k}\right)_{k=1, . ., 9}, z_{12}, z_{23}, h_{v},\right. \\
& \left.\quad \sigma_{v}, \rho_{12}, \rho_{23}, \rho_{13}\right\},
\end{aligned}
$$

where the index $n$ on $\boldsymbol{\theta}_{n}$ indicates the number of unknown parameters and $\left(t_{m, k}\right)_{k=1, \ldots, 9}$ denote the 9 unknown real coefficients which parameterize the matrix $\boldsymbol{T}_{m}$ for $m \in$ $\{v o l, g r o\}$. Note that it is possible to use symmetry properties to constrain the matrices $\boldsymbol{T}_{v o l}$ and $\boldsymbol{T}_{\text {gro }}$, and thus decrease the number of unknown parameters. However, such a priori knowledge is not useful in single-baseline [36] to improve the vegetation height estimation, and seldom used in practice [4]. Thus, we choose not to use any assumption on $\boldsymbol{T}_{\text {vol }}$ and $\boldsymbol{T}_{\text {gro }}$.

\section{B. CRB computation}

It has been shown in [35] that the CRB can be useful to characterize the estimation precision in the SB PolInSAR configuration. This paragraph summarizes the CRB properties previously presented in [35]. Consider a set $\chi=\left\{\boldsymbol{k}_{1}, \boldsymbol{k}_{2}, \ldots, \boldsymbol{k}_{N}\right\}$ of $N$ measurements of a DB PolInSAR image. Assume that the vector of unknown parameters is $\boldsymbol{\theta}=\left\{\left(\theta_{p}\right)_{p=1, \ldots, K}\right\}$ (it can be $\boldsymbol{\theta}_{22}, \boldsymbol{\theta}_{23}, \boldsymbol{\theta}_{24}$ or $\boldsymbol{\theta}_{25}$ but we removed the index to simplify notations) and consider an estimator $\widehat{\theta}_{p}(\chi)$ of the parameter $\theta_{p}$. The 
mean and variance of $\widehat{\theta}_{p}(\chi)$ are respectively denoted by $\mu_{\theta_{p}}$ and $\sigma_{\theta_{p}}^{2}$. The precision of an unbiased estimator (i.e. for which $\mu_{\theta_{p}}=\theta_{p}$ ) can be described by its variance $\sigma_{\theta_{p}}^{2}$. However, before looking for an estimator $\widehat{\theta}_{p}(\chi)$, it can be interesting to analyze $C R B_{N}\left[\theta_{p}\right]$ (i.e. the $\mathrm{CRB}$ of $\theta_{p}$ for a sample of size $N$ ), because it provides a lower bound on the variance $\sigma_{\theta_{p}}^{2}$ of unbiased estimators (i.e. $\sigma_{\theta_{p}}^{2} \geq C R B_{N}\left[\theta_{p}\right]$ ). As explained in [38], $C R B_{N}\left[\theta_{p}\right]$ is defined as the element $(p, p)$ of the inverse of the Fisher information matrix $\boldsymbol{I}_{N}(\boldsymbol{\theta})$, which is defined by $\left[\boldsymbol{I}_{N}(\boldsymbol{\theta})\right]_{(p, q) \in\{1, \ldots, 23\}^{2}}=-\left\langle\left(\partial^{2} / \partial \theta_{p} \partial \theta_{q}\right) \log P(\boldsymbol{\chi} \mid \boldsymbol{\theta})\right\rangle$, where $P(\boldsymbol{\chi} \mid \boldsymbol{\theta})$ is the probability density function of observing $\chi$ when the parameter is $\boldsymbol{\theta}$. In this paper, measurements $\boldsymbol{k}_{n}$ are assumed to be statistically independent and identically distributed with a zero mean complex circular Gaussian with covariance matrix $\Upsilon$, which means that :

$$
P(\boldsymbol{\chi} \mid \boldsymbol{\theta})=\frac{1}{\pi^{9 N} \operatorname{det}(\boldsymbol{\Upsilon})^{N}} \exp \left(-N \operatorname{tr}\left(\boldsymbol{\Upsilon}^{-1} \widehat{\boldsymbol{\Upsilon}}\right)\right)
$$

where tr is the trace, det is the determinant, $\widehat{\Upsilon}=$ $\frac{1}{N} \sum_{n=1}^{N} \boldsymbol{k}_{n} \boldsymbol{k}_{n}^{\dagger}$ is the empirical covariance matrix obtained from the measurements and $\Upsilon$ is the covariance matrix parameterized by the vector of unknown parameters $\boldsymbol{\theta}$. In which case, the element $(p, q)$ of the matrix $\boldsymbol{I}_{N}(\boldsymbol{\theta})$ can be written [42] as

$$
\left[\boldsymbol{I}_{N}(\boldsymbol{\theta})\right]_{p, q}=N \operatorname{tr}\left(\boldsymbol{\Upsilon}^{-1} \frac{\partial \boldsymbol{\Upsilon}}{\partial \theta_{p}} \boldsymbol{\Upsilon}^{-1} \frac{\partial \boldsymbol{\Upsilon}}{\partial \theta_{q}}\right),
$$

The expressions of the partial derivatives $\frac{\partial \Upsilon}{\partial \theta_{p}}$ required to calculate $C R B_{N}\left[\theta_{p}\right]$ are straightforward generalizations of those described in [35] and thus are not detailed in this paper.

Since the CRB only gives a bound of the variance of unbiased estimator, it is also important to check whether the bound is reached by an estimator. For this purpose, an iterative maximum-likelihood based estimator is proposed. The implementation of this estimator is detailed in the next section.

\section{Implemented maximum-likelihood estimator}

When the precision of vegetation height estimation in single-baseline was analyzed in [35], the Cloude et al's estimator proposed in [4] was observed to be an efficient estimator, which means that it is unbiased and its variance is equal to the CRB, when the sample size $N$ is sufficiently high. In this paper, a maximum-likelihood estimator is used in dual-baseline to provide a simple criterion taking into account all the available baselines. The maximumlikelihood estimator, previously used in [10] in singlebaseline PolInSAR configuration, is a standard algorithm which can be used in most of estimation problems (see [38] for a didactic tutorial).

In the PolInSAR literature [4], [11], [26], [24], [7], most of the estimators are based on the parametrization of coherence $\gamma_{i j}(\boldsymbol{w})=\frac{\boldsymbol{w}^{\dagger} \boldsymbol{T}_{i j} \boldsymbol{w}}{\sqrt{\boldsymbol{w}^{\dagger} \boldsymbol{T}_{i i} \boldsymbol{w}} \sqrt{\boldsymbol{w}^{\dagger} \boldsymbol{T}_{j j} \boldsymbol{w}}}$ for different pairs of polarizations described by the vector $\boldsymbol{w}$. The maximum- likelihood estimator is based on the minimization of the opposite of the log-likelihood:

$$
J(\boldsymbol{\theta})=N \ln (\operatorname{det}(\boldsymbol{\Upsilon}))+N \operatorname{tr}\left(\boldsymbol{\Upsilon}^{-1} \widehat{\boldsymbol{\Upsilon}}\right),
$$

where the covariance matrix $\Upsilon$ is parameterized by the vector $\boldsymbol{\theta}$ as previously described. The minimization of the function $J(\boldsymbol{\theta})$ allows one to estimate the unknown vector $\boldsymbol{\theta}$. In this paper, the optimization is performed with the Fisher's scoring algorithm [38], which is based on the recursive scheme:

$$
\begin{aligned}
\widehat{\boldsymbol{\theta}}_{n+1} & =\widehat{\boldsymbol{\theta}}_{n}+p_{n} \boldsymbol{d}_{n} \\
\text { with } \boldsymbol{d}_{n} & =-\left(\boldsymbol{I}_{N}\left(\widehat{\boldsymbol{\theta}}_{n}\right)\right)^{-1} \quad \boldsymbol{\nabla}_{\boldsymbol{\theta}} J\left(\widehat{\boldsymbol{\theta}}_{n}\right),
\end{aligned}
$$

where, at iteration $n$, the vector $\widehat{\boldsymbol{\theta}}_{n}$ is an estimation of the vector $\boldsymbol{\theta}$, the scalar $p_{n}$ is the step (herein chosen equal to 0.1 ), and the vector $\nabla_{\theta} J$ denotes the gradient of $J(\boldsymbol{\theta})$. The $p^{t h}$ component of $\boldsymbol{\nabla}_{\boldsymbol{\theta}} J$ is equal to $\frac{\partial J(\boldsymbol{\theta})}{\partial \theta_{p}}=$ $N\left(\operatorname{tr}\left(\boldsymbol{\Upsilon}^{-1} \frac{\partial \Upsilon}{\partial \theta_{p}}\right)-\operatorname{tr}\left(\Upsilon^{-1} \frac{\partial \Upsilon}{\partial \theta_{p}} \boldsymbol{\Upsilon}^{-1} \widehat{\Upsilon}\right)\right)$ [42]. In such an iterative procedure, it is often crucial to initialize it with a vector $\widehat{\boldsymbol{\theta}}_{0}$ not too far from the global minima of $J(\boldsymbol{\theta})$ because of the presence of local minima. A possible initialization procedure (i.e. estimation of $\widehat{\boldsymbol{\theta}}_{0}$ ), which relies on single-baseline approaches, is presented in appendix $\mathrm{A}$ and in appendix B when one estimates only one coherence $\rho$ (i.e. one uses the a priori knowledge that $\rho_{i j}=\rho$ for $i \neq j$ ). It is shown in the next section III-A that the $\mathrm{CRB}$ of the vegetation height are so large with three unknown coherences that it appeared unnecessary to implement an estimator in this case. Furthermore, with the scoring algorithm, it can also be important to regularize the inversion of the matrix $\boldsymbol{I}_{N}\left(\widehat{\boldsymbol{\theta}}_{n}\right)$ in Eq. (10) with a pseudoinverse [43] (in our implementation, the limit is when the ratio between the largest and the smallest singular value is higher than $10^{16}$ ).

Another issue is to decide when the optimization should stop. With the proposed implementation, there are 3 situations which stop the recursive scheme. The iteration stops when the quantity $\frac{1}{N}\left|J\left(\widehat{\boldsymbol{\theta}}_{n}\right)-J\left(\widehat{\boldsymbol{\theta}}_{n-1}\right)\right|$ is inferior to a given value (chosen equal to $10^{-6}$ in this paper), or when the number of iterations $n_{\max }=1000$ is reached, or when the optimization algorithm is providing parameter values leading to a non-defined criterion value $J\left(\widehat{\boldsymbol{\theta}}_{n}\right)$. This last situation usually occurs because the estimated covariance matrix $\widehat{\boldsymbol{T}}_{\text {vol }}$ or $\widehat{\boldsymbol{T}}_{\text {gro }}$ has negative eigenvalues. In this case, the estimator is considered to fail. In addition, if the obtained value $\widehat{h}_{v}$ is with an error $\left|\widehat{h}_{v}-h_{v}\right|$ larger than $6 \mathrm{~m}$, then the estimator is also considered to fail. This arbitrary value of $6 \mathrm{~m}$ allows us to define outliers (i.e. bad estimates), whose proportion is analyzed in the simulation section with the so-called estimated "success rate".

\section{Simulated RVoG model parameters}

A straightforward adaptation of the result obtained in [37] for the single-baseline case, to the dual-baseline configuration shows that the $\mathrm{CRB}$ of the vegetation height and the variance of the maximum likelihood estimator depends 
on the form of $\boldsymbol{T}_{v o l}$ and $\boldsymbol{T}_{\text {gro }}$ only through the 3 eigenvalues of $\boldsymbol{T}_{v o l}^{-1} \boldsymbol{T}_{\text {gro }}$ denoted $\lambda_{n}$ (with $\lambda_{1}>\lambda_{2}>\lambda_{3}$ ). In other words all datasets for which $\boldsymbol{T}_{v o l}^{-1} \boldsymbol{T}_{\text {gro }}$ are equal to $\left(\lambda_{n}\right)_{n=1,2,3}$ (with identical values for $h_{v}, \sigma_{v}, \rho, \ldots$ ) have the same vegetation height estimation precision. Thus, in the simulations of this paper, we generated synthetic data with

$$
\boldsymbol{T}_{\text {vol }}=\left(\begin{array}{ccc}
1 & 0 & 0 \\
0 & 1 & 0 \\
0 & 0 & 1
\end{array}\right) \quad \boldsymbol{T}_{\text {gro }}=\left(\begin{array}{ccc}
\lambda_{1} & 0 & 0 \\
0 & \lambda_{2} & 0 \\
0 & 0 & \lambda_{3}
\end{array}\right)
$$

Furthermore, the adaptation of [37] in dual-baseline also shows that the vegetation height estimation precision does not depend on the values of $z_{12}$ and $z_{23}$ (even in the situation with $z_{12} \neq z_{23}$ ). Thus, in the simulations of this paper, we generated synthetic data with

$$
z_{12}=z_{23}=1 \mathrm{~m}
$$

The parameters that influence the precision of vegetation height estimation are $\beta, k_{12}^{z}, k_{23}^{z}, h_{v}, \sigma_{v}, \rho$ and $\left(\lambda_{n}\right)_{n=1,2,3}$. Exploring the whole space of dimension 9 is not possible. In section III, first, the variation of the CRB of $h_{v}$ as a function of $k_{12}^{z}, k_{23}^{z}$ is analyzed, to discuss the potential optimization of the system. Next, the variation of the CRB of $h_{v}$ is analyzed as a function of $h_{v}, \sigma_{v}$, $\rho, \mathcal{A}=\left(\lambda_{1}-\lambda_{3}\right) /\left(\lambda_{1}+\lambda_{3}\right)$ and $\mathcal{E}=\lambda_{1}+\lambda_{2}+\lambda_{3}$. As explained in [37], the parameter $\mathcal{A}$ is related to the difference between the polarimetric properties of $\boldsymbol{T}_{v o l}$ and those of $\boldsymbol{T}_{\text {gro }}$. On the one hand, $\mathcal{A}=0$ implies that $\boldsymbol{T}_{\text {vol }}$ and $\boldsymbol{T}_{\text {gro }}$ are proportional and coherence $\gamma_{i j}(\boldsymbol{w})$ are independent of $\boldsymbol{w}$. On the other hand, $\mathcal{A}=1$ implies that a pair of polarization exists, described by a vector $\boldsymbol{w}$, for which the ground response contribution is null (i.e. for which $\left.\gamma_{i j}(\boldsymbol{w})=e^{\mathrm{j} k_{i j}^{z} z_{i j}} \frac{\rho_{i j} I_{i j}}{\sqrt{I_{i i} I_{j j}}}\right)$. The parameter $\mathcal{E}$ is related to the ground-to-volume power ratio between the wave backscattered by the ground and the one backscattered by the volume.

The relation between $\left(\lambda_{n}\right)_{n=1,2,3}$ and $\mathcal{A}, \mathcal{E}, \mathcal{X}$ is given by

$$
\begin{aligned}
\lambda_{1} & =\mathcal{E}(1+\mathcal{A}) /(3-\mathcal{A}+2 \mathcal{A} \mathcal{X}) \\
\lambda_{2} & =\mathcal{E}(1-\mathcal{A}+2 \mathcal{A} \mathcal{X}) /(3-\mathcal{A}+2 \mathcal{A} \mathcal{X}) \\
\lambda_{3} & =\mathcal{E}(1-\mathcal{A}) /(3-\mathcal{A}+2 \mathcal{A} \mathcal{X})
\end{aligned}
$$

where $\mathcal{X}=\left(\lambda_{2}-\lambda_{3}\right) /\left(\lambda_{1}-\lambda_{3}\right)$.

\section{PRECISION ANALYSIS}

\section{A. Evolution of the CRB as a function of the system configuration}

Figure 1 shows the evolution of the CRB of $h_{v}$ as a function of the system configuration for different a priori knowledge (see Eq. (6)) and for $N=200$ pixels. For all considered a priori knowledge, when $k_{12}^{z}=2 \pi n / h_{v}$ and $k_{23}^{z}=2 \pi m / h_{v}$ with $(n, m)$ a pair of strictly positive integers, the precision described by $C R B_{N}^{1 / 2}\left(h_{v}\right)$ becomes larger than $10 \mathrm{~m}$ (these particular values are denoted by white dashed lines in Figure 1(a-d)). Thus, for these values, it is impossible to have an accurate estimation of $h_{v}$. In the rest of the space $\left(k_{12}^{z}, k_{23}^{z}\right)$, the value of $C R B_{N}^{1 / 2}\left(h_{v}\right)$ remains larger than $6 \mathrm{~m}$ in most cases where 3 temporal coherences are estimated (see (a) and (b)). Nevertheless, the value of $C R B_{N}^{1 / 2}\left(h_{v}\right)$ often lies below $2 \mathrm{~m}$ when one estimates only one temporal coherence (see (c) and (d)). When the vegetation height $h_{v}$ varies in the interval $[10 \mathrm{~m}, 50 \mathrm{~m}]$, similar images will be obtained and the patterns centered at $k_{12}^{z}=2 \pi n / h_{v}$ and $k_{23}^{z}=2 \pi m / h_{v}$ will partly fill the space $\left(k_{12}^{z}, k_{23}^{z}\right)$. Thus, it seems impossible to estimate the vegetation height accurately when 3 temporal coherences are unknown. For the rest of the paper, we thus focus on situations (c) and (d), for which only one temporal coherence $\rho$ is estimated. Moreover, based on Figure 1, we choose $k_{12}^{z}=0.06 \mathrm{~m}^{-1}$ and $k_{23}^{z}=0.25 \mathrm{~m}^{-1}$ so that the $\mathrm{CRB}$ of $h_{v}$ remains small for $h_{v} \in[10,50] \mathrm{m}$.

Furthermore, figure 1 (c) and (d) show that knowing a priori that $z_{12}=z_{23}$ can also allow the reduction of the CRB of $h_{v}$ compared to the case where this a priori is unknown. In the following, we continue to compare these two cases. In the next paragraph, the proposed maximum likelihood estimator is thus analyzed in both scenarios where $\boldsymbol{\theta}_{22}$ or $\boldsymbol{\theta}_{23}$ are estimated.

\section{B. Evolution of the $C R B$ and of the proposed estimator performances as a function of $h_{v}$ and $\mathcal{A}$}

This section presents an analysis of the performances of the estimators obtained with Monte-Carlo simulations. For each example considered, $P=200$ realisations of the sample $\chi^{(p)}=\left\{\boldsymbol{k}_{1}^{(p)}, \boldsymbol{k}_{2}^{(p)}, \ldots, \boldsymbol{k}_{N}^{(p)}\right\}$ (with $p \in\{1,2, \ldots, P\}$ ) have been generated according to the considered model (see Eqs. (1) and (2)) assuming a Gaussian circular distribution. Then, the empirical root mean square errors (RMSE) $\widehat{r m s e} \widehat{h}_{v}^{2}=\frac{1}{P} \sum_{p}\left(\widehat{h}_{v}\left(\chi^{(p)}\right)-h_{v}\right)^{2}$ are computed. One also could have analyzed bias and variance separately, but we preferred to add these two sources of error and concentrate on the presence of outliers with the analysis of the percentage of success of the estimator. The size of sliding windows in PolInSAR images is usually around 200 pixels, we choose to analyze the performances for $N=200$ and $N=2000$ pixels. Furthermore, the iterative estimator performance are analyzed for two cases:

- When the initial estimation $\widehat{\boldsymbol{\theta}}_{0}$ is obtained with the algorithm presented in Appendix A.

- When the initial estimation $\widehat{\boldsymbol{\theta}}_{0}$ is fixed to the true vector $\boldsymbol{\theta}$.

Obviously this second initialization cannot be used with real data. The purpose is to analyze the performance of the estimator when the iterative estimator is initialized near the solution and thus is less sensitive to local maxima.

The results of the Monte Carlo simulation presented in Figure 2 (a) and (b) show that the percentage of success is always close to $100 \%$ when the initialization is at the solution, but it is not the case with the initialization proposed in Appendix A. When $\mathcal{A}=0.3$ (i.e. small polarization contrast) and $h_{v}=28 \mathrm{~m}$, these pourcentages are around $60 \%$ for both estimator (a) and (b). 

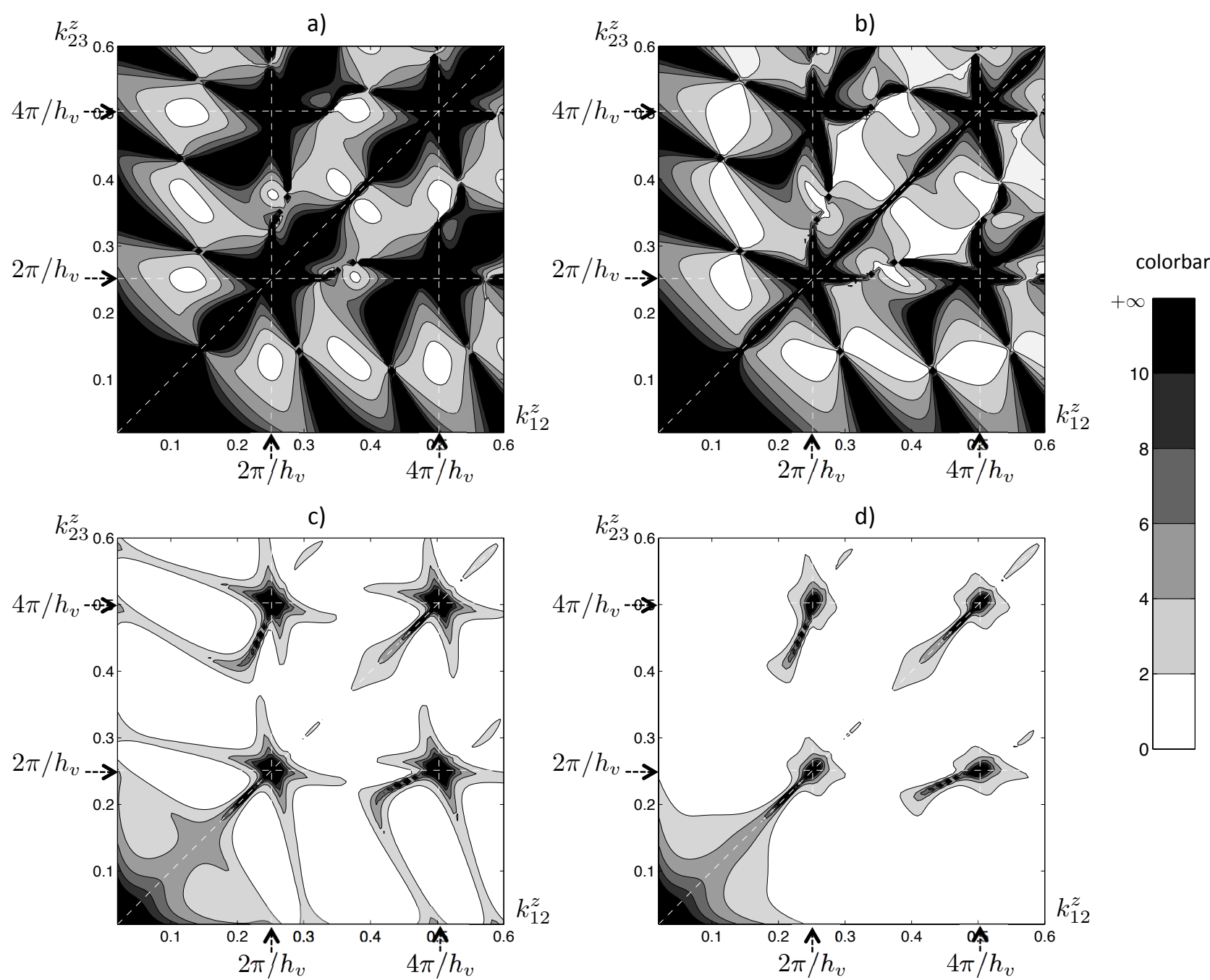

Fig. 1: Evolution of the vegetation height $\mathrm{CRB}$ as a function of $k_{12}^{z}, k_{23}^{z}$ for $N=200$ pixels, $\mathcal{A}=0.3, \mathcal{E}=800 \mathrm{~m}^{-1}$, $\mathcal{X}=0.2, \beta=35^{\circ}, \sigma_{v}=0.023 \mathrm{~m}^{-1}, \rho=0.8$ and $h_{v}=25 \mathrm{~m}$. (a) $C R B_{N}^{1 / 2}\left(h_{v}\right)$ for $\boldsymbol{\theta}_{25}$ (i.e. 3 estimated temporal coherences and 2 estimated ground heights). (b) $C R B_{N}^{1 / 2}\left(h_{v}\right)$ for $\boldsymbol{\theta}_{24}$ (i.e. 3 estimated temporal coherences and 1 estimated ground height). (c) $C R B_{N}^{1 / 2}\left(h_{v}\right)$ for $\boldsymbol{\theta}_{23}$ (i.e. 1 estimated temporal coherence and 2 estimated ground heights). (d) $C R B_{N}^{1 / 2}\left(h_{v}\right)$ for $\boldsymbol{\theta}_{22}$ (i.e. 1 estimated temporal coherence and 1 estimated ground height).

Furthermore, Figure 2 (c) and (d) show that $C R B_{N}^{1 / 2}\left(h_{v}\right)$ varies a lot with $h_{v}$, and in particular that $C R B_{N}^{1 / 2}\left(h_{v}\right)$ increases when the vegetation height is smaller than $15 \mathrm{~m}$. Thus, the chosen pair of vertical wavenumber is not adequate for small vegetations. Moreover, a comparison between Figure 2 (c) and (d) shows that $C R B_{N}^{1 / 2}\left(h_{v}\right)$ can be bigger when two ground heights are unknown instead of one. For $\mathcal{A}=0.3$ and $h_{v}=30 \mathrm{~m}$, the $C R B_{N}^{1 / 2}\left(h_{v}\right)$ increases from $0.7 \mathrm{~m}$ (in d) to $2 \mathrm{~m}$ (in c). It seems that the influence of the polarimetric contrast $\mathcal{A}$ on $C R B^{1 / 2}\left(h_{v}\right)$ remains modest when only one ground height is unknown, whereas it is not the case when two ground heights are unknown.

Figure 2 (c) and (d) also show that the root mean square error (RMSE) computed with estimations with an error smaller than $6 \mathrm{~m}$ are close to the precision given by $C R B^{1 / 2}\left(h_{v}\right)$, especially for large values of $\mathcal{A}$. Thus, the main issue of the estimator is the amount of outliers described by the pourcentage of success. These results are confirmed by Figure 3, where simular observations are obtained for $\mathcal{A}=0.1$ and $\mathcal{A}=0.6$.

The problem that occur for $\mathcal{A}=0.1$ with the initialization proposed in Appendix A (see the pourcentage of success smaller than $60 \%$ in Figure 3(a) and (b)) probably comes from the inaccuracy of single-baseline estimations [37]. In such situations, one alternative is to increase the number of pixels. Figure 4 shows that when $N=2000$, the pourcentage of success increases compared to the situation with $N=200$. However, this supposes one can consider large homogeneous region of vegetation in the considered image.

To summarize, the precision given by the CRB is encouraging, but the proposed estimators may not be efficient when $\mathcal{A}$ is too small. Finding some optimization algorithms to obtain a better estimator is a motivating perspective. Another solution is to increase the window 

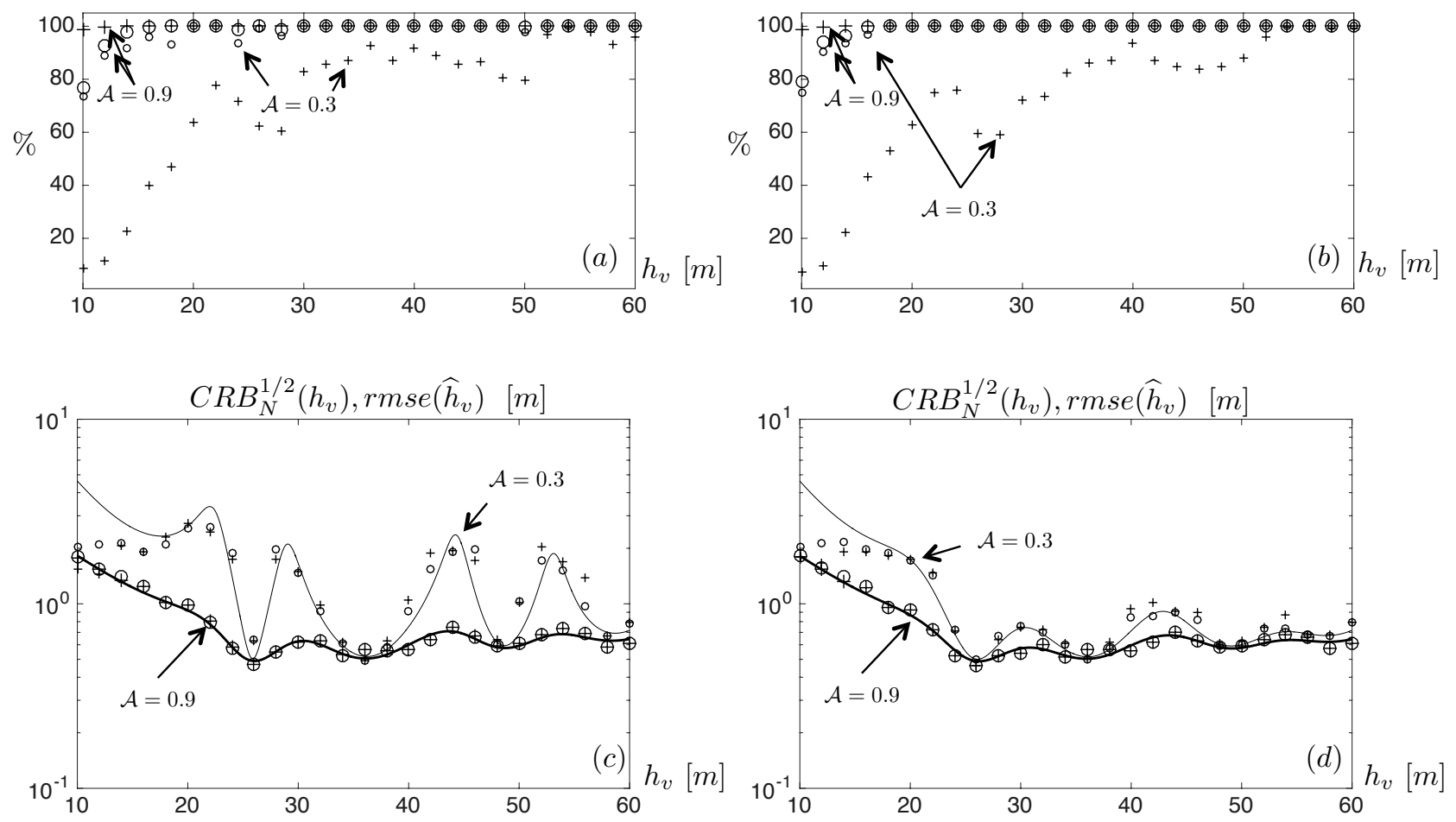

Fig. 2: Performances of the vegetation height estimators, as a function of $h_{v}$ and for different values of $\mathcal{A}(\mathcal{A}=0.3$ and $\mathcal{A}=0.9$ ). In (a) and (c), estimations have been obtained when 2 ground heights are estimated (i.e. $\boldsymbol{\theta}_{23}$ in Eq. (6)), whereas in (b) and (d), estimations have been obtained when only 1 ground height is estimated (i.e. $\boldsymbol{\theta}_{22}$ in Eq. (6)). In (a) and (b) the success rate indicates the percentage of $\widehat{h}_{v}$ estimations with an error smaller than $6 \mathrm{~m}$. In (c) and (d) Root mean square errors (RMSE) are computed with estimations for which the error is smaller than 6 m. Straight lines are $C R B_{N}^{1 / 2}\left(h_{v}\right)$, the sign + stands for estimations obtained with the scoring algorithm when the initial estimations $\widehat{\boldsymbol{\theta}}_{0}$ are given by the algorithm described in appendix A, and circle $o$ stands for the one obtained when $\widehat{\boldsymbol{\theta}}_{0}$ is fixed to the true value. Large + and $o$ are obtained for $\mathcal{A}=0.9$ and small ones for $\mathcal{A}=0.3$. The other parameters are: $k_{12}^{z}=0.06 \mathrm{~m}^{-1}, k_{23}^{z}=0.25 \mathrm{~m}^{-1}, N=200$ pixels, $\mathcal{E}=800 \mathrm{~m}^{-1}, \mathcal{X}=0.2, \beta=35^{\circ}, \sigma_{v}=0.023 \mathrm{~m}^{-1}$ and $\rho=0.8$.
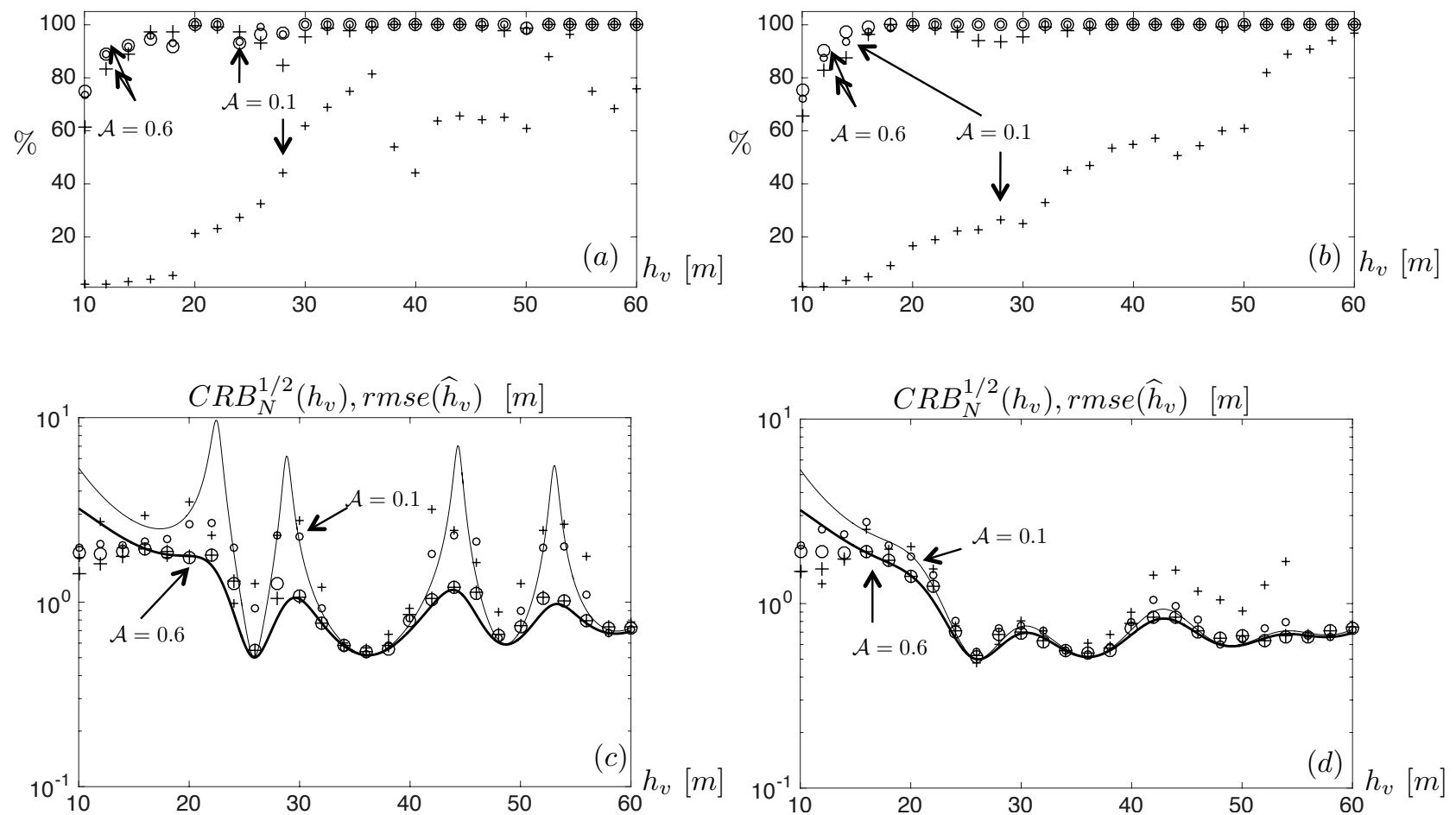

Fig. 3: Same as Figure 2, but for $\mathcal{A}=0.6$ (large crosses and circles) and $\mathcal{A}=0.1$ (small crosses and circles). 

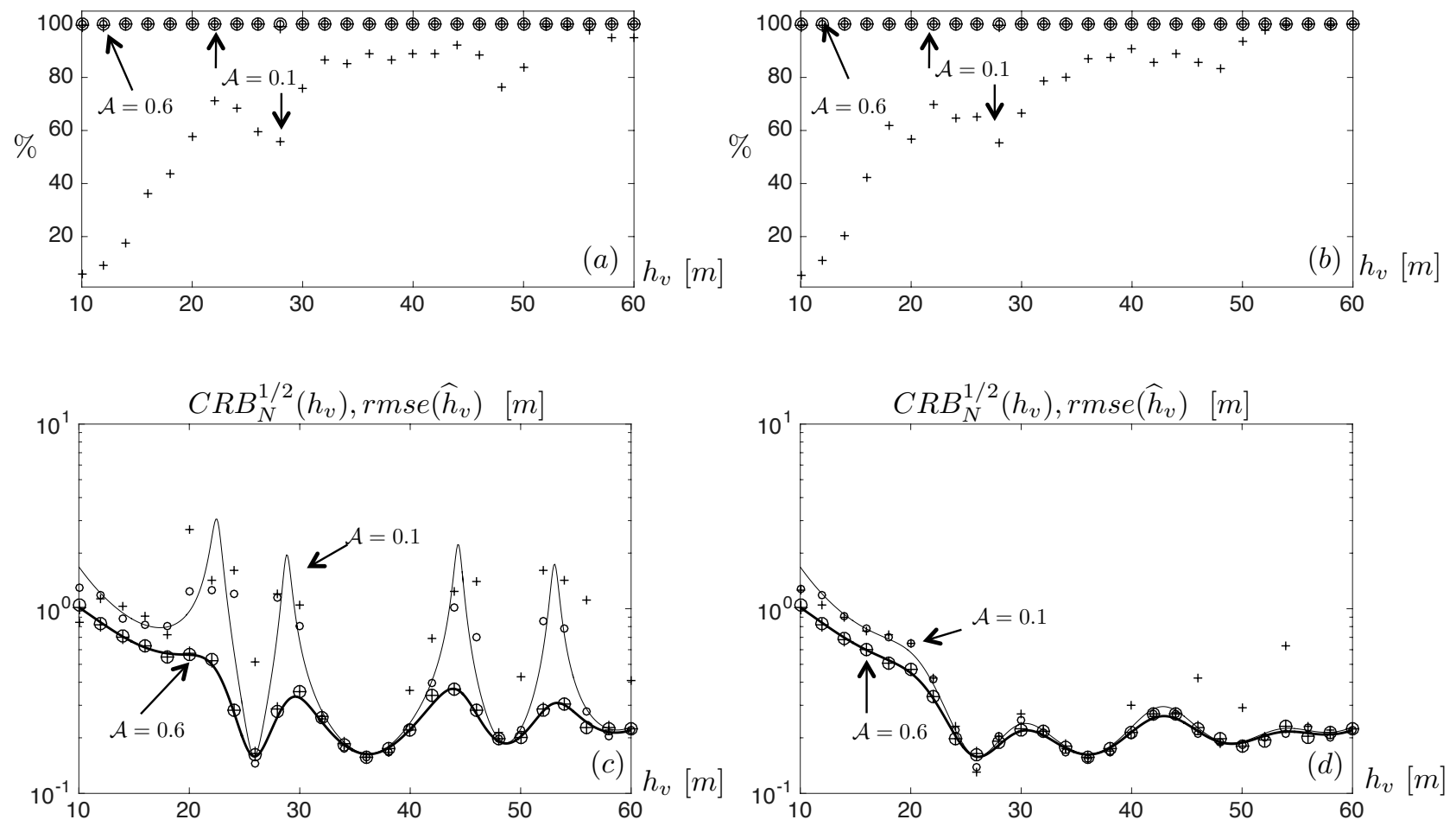

Fig. 4: Same as Figure 3, but for $N=2000$ pixels.

size, however this solution can lead to inhomogeneous samples. Thus, the analysis of the estimator performance in presence of inhomogeneities should be explored to analyze the bias induced by these inhomogeneities. These two aspects are not in the scope of this paper but open challenging perspectives. In the following, we choose $\mathcal{A}=0.3$ and investigate the influence of other vegetation parameters on $C R B^{1 / 2}\left(h_{v}\right)$.

\section{Influence of other vegetation parameters}

In this paper, we propose a methodology to analyze the precision that can be expected on the vegetation height estimation with dual-baseline configuration and with RVoG model without knowing a priori the extinction coefficient $\sigma_{v}$ and in presence of an unknown temporal decorrelation of the volume (described herein by a single coherence parameter $\rho$ ). The previously presented results show that the precision described by the square root of the CRB varies significantly as a function of $h_{v}$. Investigating the physical reasons of these variations is a major issue that require further investigations. In the following, we analyse the influence of different parameters on the CRB: the parameter $\mathcal{E}$, the attenuation $\sigma_{v}$ and the temporal coherence parameter $\rho$.

Figure 5 shows that when two ground heights are unknown, some local maxima appear at several values of $h_{v}$. The intensity and the location of these local maxima change with $\mathcal{E}$ and $\sigma_{v}$. When data are calibrated such that $z_{12}=z_{23}$, the importance of these local maxima is reduced significantly. This observation is in favor of calibrating the data phases before estimating $h_{v}$.
Furthermore, an increase from $\mathcal{E}=50 \mathrm{~m}^{-1}$ to $\mathcal{E}=$ $200 \mathrm{~m}^{-1}$ (i.e. an increase of the ground-to-volume power ratio of a factor 4) make the CRB of $h_{v}$ decrease for $h_{v}>30 \mathrm{~m}$, but an increase from $\mathcal{E}=200 \mathrm{~m}^{-1}$ to $\mathcal{E}=2000 \mathrm{~m}^{-1}$ seems to induce some CRB increases, especially with two unknown ground heights. This can be interpreted as a tradeoff on the ground-to-volume energy ratio. For the extinction coefficient $\sigma_{v}$, it seems that the lowest CRB occurs when $\sigma_{v} \in[0.023,0.046]$. When $\sigma_{v}=0.069 \mathrm{~m}^{-1}$, the vegetation height CRB increase rapidly after $50 \mathrm{~m}$, probably because the ground is not visible anymore. Note that these phenomena also depend on $\mathcal{A}$ and on the other parameters. For temporal coherence $\rho$, the situation looks simpler. For $h_{v} \leq 60 \mathrm{~m}$, the CRB seem to be approximately a function of $1 / \rho$.

\section{COnClusion And Future Work}

We proposed a methodology to analyze the precision on the vegetation height estimation using the PolInSAR system in the DB configuration with an RVoG model, where the temporal decorrelation on the volume is unknown and where there is no a priori knowledge on the extinction coefficient. The influence of the system configuration and vegetation parameters on the value of the vegetation height CRB shows that using an RVoG model with three unknown temporal coherences seems hazardous, since the obtained precision on the vegetation height is usually larger than $6 \mathrm{~m}$ for $N=200$ pixels. Even with only one unknown temporal coherence, the precision on the vegetation height estimation varies widely as a function of $h_{v}$ and the other vegetation characteristics. For the considered simulations, the precision varies from 0.5 to $10 \mathrm{~m}$ for a sample size 

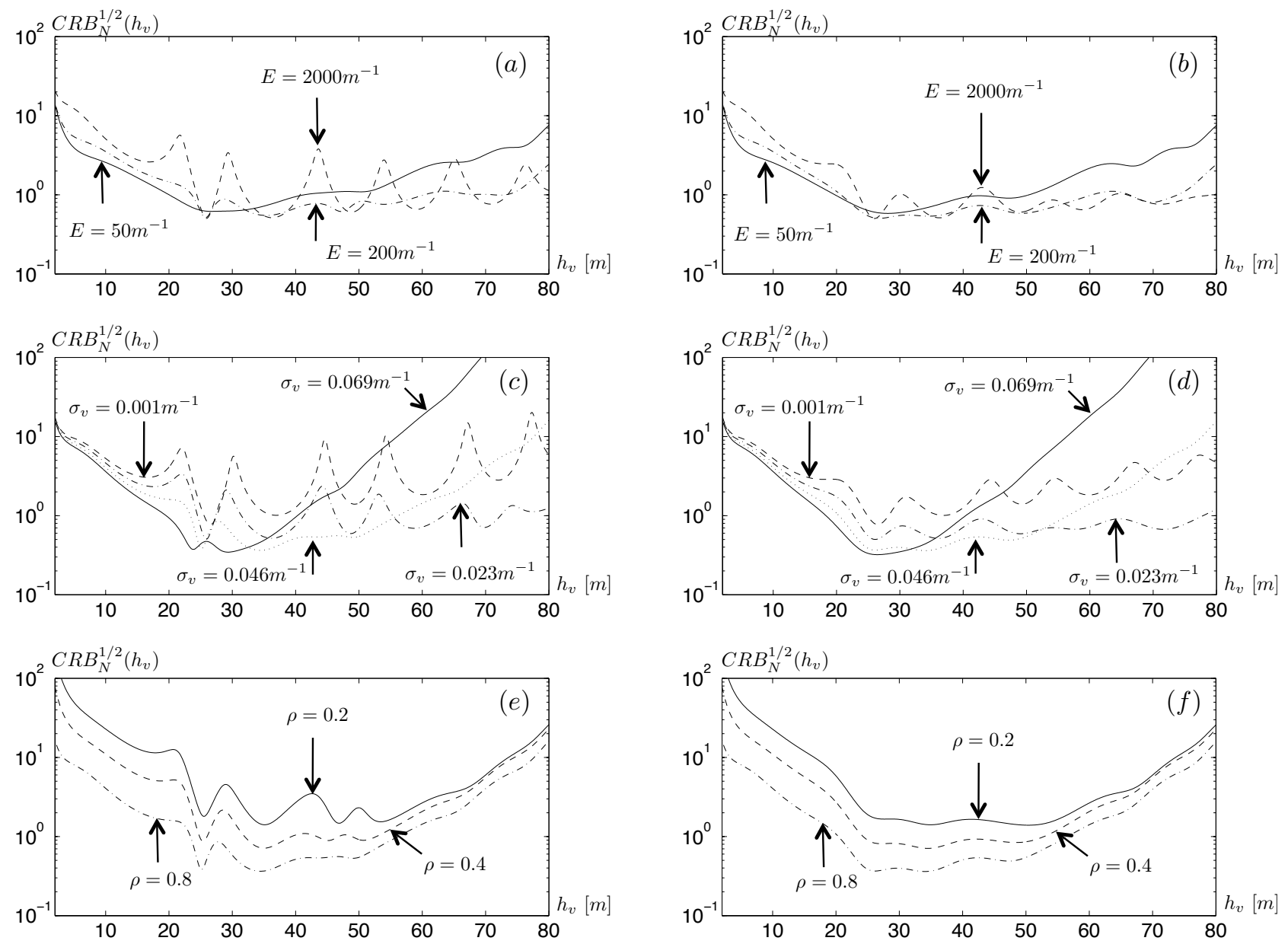

Fig. 5: Evolution of the CRB with $\mathcal{E}$ (a and b), with $\sigma_{v}$ (c and d) and with $\rho$ (e and f). On the left (a, c and e), two ground heights are unknown. On the right (c, d and $\mathrm{f}$ ), one ground height is unknown. Unless otherwise specified, parameters are $k_{12}^{z}=0.06 \mathrm{~m}^{-1}, k_{23}^{z}=0.25 \mathrm{~m}^{-1}, N=200$ pixels, $\mathcal{A}=0.3, \mathcal{E}=800 \mathrm{~m}^{-1}, \mathcal{X}=0.2, \beta=35^{\circ}$, $\sigma_{v}=0.023 \mathrm{~m}^{-1}$ and $\rho=0.8$.

of 200 pixels. The observed sensitivity of the vegetation height $\mathrm{CRB}$ to $\mathcal{A}, \mathcal{E}$ and $h_{v}$ shows that the description of the problem is complex in dual-baseline. The presented comparison between situations where one or two ground heights are unknown, shows that the difference of performance can remain small. Thus, with phase-calibrated data, it makes sense to use the two proposed estimators and compare their results.

For the analyzed examples, the proposed maximum likelihood estimators seem to be efficient for a sample size of 200 pixels when the polarimetric contrast is larger than 0.6 , but often leads to outliers when the polarimetric contrast is smaller than 0.3. This problem may occur because of the initial estimations and therefore the optimization process could be improved. The presented simulations also show that CRBs vary drastically with respect to the ground-to-volume power ratio (from 1 to $5 \mathrm{~m}$ ), the extinction coefficient (from 1 to $10 \mathrm{~m}$ ) and the temporal coherence. An obvious perspective is to apply the proposed maximum-likelihood (ML) estimators to real data sets to analyze whether the considered simple model with only one temporal coherence coefficient could be well-suited to extract information from real data with dualbaseline configuration. Since the ML estimator provide estimations of all RVoG parameters, it will be possible to analyze the estimated values of the extinction, the ground heights, and the polarimetric coherency matrices relative to the volume and to the ground. Simultaneously, it will be important to analyse the potential precision of these estimations with the CRB. Another promising perspective is to apply the proposed methodology to other models. In particular, one could investigate the potential of a relatively large number of PolInSAR measurements for biomass parameter estimation, when these are available, to analyze whether such multi-baseline configurations can significantly outperform single-baseline and dual-baseline approaches.

\section{APPENDIX}

\section{A. Estimation of $\widehat{\boldsymbol{\theta}}_{0}$}

The algorithm which allows one to get an initial estimation $\widehat{\boldsymbol{\theta}}_{0}$ is summarized on Figure 6 . It consists in exploring the two-dimensional (2D) space formed by the parameters $\rho$ and $\sigma_{v}$. For each pair of available baseline, 
one assumed that $\rho$ and $\sigma_{v}$ are known and one uses singlebaseline approaches [4] to estimate potential values of $\boldsymbol{\theta}$. Once the whole range of $\rho$ and $\sigma_{v}$ has been covered, the best estimation according to the dual-baseline likelihood criterion is retained. In the considered implementation of this algorithm, the parameter $\rho$ is assumed in the range $[0.1,1]$ and the parameter $\sigma_{v}$ is assumed in the range $\left[10^{-5} \mathrm{~m}^{-1}, 10^{-1} \mathrm{~m}^{-1}\right]$. Because implementing a loop with 1000 values for each parameter $\left(\rho\right.$ and $\sigma_{v}$ ) is time consuming, we choose to implement 3 successive loops with only 11 values for each parameter to accelerate the process. The detail of these different steps follows.

loop on $\rho$ and $\sigma_{v}$

$\left(\widetilde{\rho}, \widetilde{\sigma}_{v}\right)$

$$
\forall(i, j) \in\{(1,2),(1,3),(2,3)\}
$$

single-baseline estimation $\widehat{\boldsymbol{\theta}}_{i, j}$

( $\rho$ and $\sigma_{v}$ assumed to be known)

$$
\begin{aligned}
& \left(\widehat{\boldsymbol{\theta}}_{i, j}\right)_{(i, j) \in\{(1,2),(1,3),(2,3)\}} \\
& \widehat{\boldsymbol{\theta}}\left(\widetilde{\rho}, \widetilde{\sigma}_{v}\right)=\arg \min _{i, j} J\left(\widehat{\boldsymbol{\theta}}_{i, j}\right) \\
& \left(\widehat{\boldsymbol{\theta}}\left(\widetilde{\rho}, \widetilde{\sigma}_{v}\right)\right)_{\left(\widetilde{\rho}, \widetilde{\sigma}_{v}\right)} \\
& \widehat{\boldsymbol{\theta}}=\arg \min _{\widetilde{\rho}, \widetilde{\sigma_{v}}} J\left(\widehat{\boldsymbol{\theta}}\left(\widetilde{\rho}, \widetilde{\sigma}_{v}\right)\right)
\end{aligned}
$$

Fig. 6: Flow chart of the initialization algorithm, which consists in finding the best single-baseline solution with a double loop on the unknown temporal coherence $\rho$ and on the extinction coefficient $\sigma_{v}$. The criterion $J$ is the dualbaseline likelihood (see Eq. (9))

Firstly, for each baseline $i j$ (with $(i, j) \in$ $\{(1,2),(2,3),(1,3)\})$, introducing $\left(\widehat{\boldsymbol{T}}_{i j}\right)_{\left\{(i, j) \in\{1,2,3\}^{2}\right\}}$ as the empirical covariance matrices, potential ground heights $z^{S B i j}$ are estimated using the single-baseline (SB) Cloude estimator [4]. The two intersections in the complex plane between a least square line fit of the three eigenvalues of the contraction matrix $\widehat{\boldsymbol{\Pi}}_{i j}=\left(\widehat{\boldsymbol{T}}_{i i}\right)^{-1 / 2} \widehat{\boldsymbol{T}}_{i j}\left(\widehat{\boldsymbol{T}}_{j j}\right)^{-1 / 2}$ [44] and the unit circle provide two candidates $\phi_{1}^{S B i j}$ and $\phi_{2}^{S B i j}$ for the ground phase of the baseline $i j$. Clearly, this first step provides several possible estimations of the ground heights. In particular, for $\boldsymbol{\theta}_{22}$, there are six available solutions (2 for each pair of baseline), whereas for $\boldsymbol{\theta}_{23}$, there are the 8 possible ground height combinations that satisfy equation (4). To remove this ambiguity, one estimate $\boldsymbol{\theta}$ for all potential candidates (as detailed in the next paragraph), and finally keep the vector $\boldsymbol{\theta}$ that minimize the dual-baseline likelihood criterion $J(\boldsymbol{\theta})$ (see Eq. (9)). This criterion is able to remove the ambiguity because it takes into account all baselines.
Secondly, we use a 2D grid on the extinction $\sigma_{v}$ and on the temporal coherence coefficient $\rho$, considering 11 potential values $\widetilde{\sigma}_{v}$ in the range $\left[10^{-5}, 10^{-1}\right]\left[\mathrm{m}^{-1}\right]$ and 11 potential values $\widetilde{\rho}$ in the range $[0.1,1.0]$, and we compute the criterion $J(\boldsymbol{\theta})$ for each considered pair $\left(\widetilde{\sigma}_{v}, \widetilde{\rho}\right)$. Indeed, for each pair $\left(\widetilde{\sigma}_{v}, \widetilde{\rho}\right)$, the estimation of the intersections between the complex function

$$
\begin{aligned}
\widetilde{\gamma}_{i j}(h) & =\widetilde{\rho} \frac{e^{\mathrm{j} k_{i j}^{z} h}-e^{-\widetilde{\alpha} h}}{1-e^{-\widetilde{\alpha} h}} \frac{\widetilde{\alpha}}{\mathrm{j} k_{i j}^{z}+\widetilde{\alpha}} \\
\text { with } \widetilde{\alpha} & =2 \widetilde{\sigma}_{v} / \cos \beta
\end{aligned}
$$

and the previous line fit (rotated by $k_{i j}^{z} z^{S B i j}$ ) provides either zero, one or two candidate vegetation heights, denoted by $\widetilde{h}_{v}^{i j}$ for each baseline $i j$. When this process leads to two candidates, the ambiguity is removed by keeping the one that minimizes the criterion $J(\boldsymbol{\theta})$. Coherency matrices $\widetilde{\boldsymbol{T}}_{\text {vol }}^{i j}$ and $\widetilde{\boldsymbol{T}}_{\text {gro }}^{i j}$ are estimated as detailed in appendix B. After parameters have been estimated for each baseline $i j$, the estimation that minimizes the criterion $J(\boldsymbol{\theta})$ (see Eq. (9)) is retained. Furthermore, once the whole 2D grid on $\sigma_{v}$ and $\rho$ has been covered, the estimation that minimizes the criterion $J(\boldsymbol{\theta})$ is retained.

Next, to improve the accuracy of these estimations, a finer $2 \mathrm{D}$ grid, containing new $11 \times 11$ pairs $\left(\widetilde{\sigma}_{v}, \widetilde{\rho}\right)$ but centered on these previous estimations of $\left(\sigma_{v}, \rho\right)$ is used. This refinement of the $2 \mathrm{D}$ grid is applied a third and last time.

\section{B. Estimation of $\boldsymbol{T}_{\text {vol }}$ and $\boldsymbol{T}_{\text {gro }}$}

This section details the estimation of the coherency matrix parameters $\left(\widetilde{t}_{v o l, k}^{i j}\right)_{k=1, \ldots, 9}$ and $\left(\widetilde{t}_{\text {gro,k }}^{i j}\right)_{k=1, \ldots, 9}$ from $\widehat{\boldsymbol{T}}_{i i}, \widehat{\boldsymbol{T}}_{j j}$ and $\widehat{\boldsymbol{T}}_{i j}$ when the parameters $z^{S B i j}, \widetilde{h}_{v}^{i j}, \widetilde{\sigma}_{v}$ and $\widetilde{\rho}$ are already fixed. Similarly to [9], the coherency matrix estimation problem can be reformulated from equation (2) and from equation (5) through the following equations:

$$
\begin{aligned}
\boldsymbol{T}_{i i}=\boldsymbol{T}_{j j} & =\boldsymbol{T}_{v}+\boldsymbol{T}_{g} \\
\boldsymbol{T}_{i j} & =e^{\mathrm{j} k_{i j}^{z} z_{i j}}\left(\rho \gamma_{v} \boldsymbol{T}_{v}+\boldsymbol{T}_{g}\right) \quad \text { if } i \neq j,
\end{aligned}
$$

where the matrices $\boldsymbol{T}_{v}$ and $\boldsymbol{T}_{g}$ and the ratio $\gamma_{v}$ are defined by:

$$
\begin{aligned}
\boldsymbol{T}_{v} & =I_{1} \boldsymbol{T}_{\text {vol }} \quad \text { with } \quad I_{1}=I_{i i}=\frac{1-e^{-\alpha h_{v}}}{\alpha} \\
\boldsymbol{T}_{g} & =a \boldsymbol{T}_{g r o} \\
\gamma_{v} & =\frac{I_{i j}}{I_{1}}=\frac{e^{\mathrm{j} k_{i j}^{z} h_{v}}-e^{-\alpha h_{v}}}{1-e^{-\alpha h_{v}}} \frac{\alpha}{\mathrm{j} k_{i j}^{z}+\alpha} .
\end{aligned}
$$

The estimated matrices $\widetilde{\boldsymbol{T}}_{\text {vol }}^{i j}$ and $\widetilde{\boldsymbol{T}}_{\text {gro }}^{i j}$ can be thus deduced from: Â£

$$
\begin{aligned}
& \widetilde{\boldsymbol{T}}_{\text {vol }}^{i j}=\frac{1}{\widetilde{I}_{1}} \widetilde{\boldsymbol{T}}_{v} \text { with } \widetilde{I}_{1}=\frac{1-e^{-\widetilde{\alpha} \tilde{h}_{v}^{i j}}}{\widetilde{\alpha}} \text { and } \widetilde{\alpha}=2 \widetilde{\sigma}_{v} / \cos \beta \\
& \widetilde{\boldsymbol{T}}_{\text {gro }}^{i j}=\frac{1}{\widetilde{a}} \widetilde{\boldsymbol{T}}_{g} \text { with } \widetilde{a}=e^{-\widetilde{\alpha} \widetilde{h}_{v}^{i j}},
\end{aligned}
$$


where the matrices $\widetilde{\boldsymbol{T}}_{v}$ and $\widetilde{\boldsymbol{T}}_{g}$ are determined using:

$$
\begin{aligned}
\boldsymbol{W} & =e^{-\mathrm{j} k_{i j}^{z} z^{S B i j}} \widehat{\boldsymbol{T}}_{i j} \\
\boldsymbol{T} & =\frac{1}{2}\left(\widehat{\boldsymbol{T}}_{i i}+\widehat{\boldsymbol{T}}_{j j}\right) \\
\boldsymbol{A} & =\boldsymbol{T}-\frac{1}{2}\left(\boldsymbol{W}+\boldsymbol{W}^{\dagger}\right) \\
\widetilde{\gamma}_{v} & =\frac{e^{\mathrm{j} k_{i j}^{z} \tilde{r}_{v}^{j}}-e^{-\widetilde{\alpha} \widetilde{h}_{v}^{i j}}}{1-e^{-\widetilde{\alpha} \widetilde{h}_{v}^{i j}}} \frac{\widetilde{\alpha}}{\mathrm{j} k_{i j}^{z}+\widetilde{\alpha}} \\
\widetilde{\boldsymbol{T}}_{v} & =\frac{\boldsymbol{A}}{1-\Re\left(\widetilde{\widetilde{\alpha}} \widetilde{\widetilde{T}}_{v}\right)} \\
\widetilde{\boldsymbol{T}}_{g} & =\boldsymbol{T}-\widetilde{\boldsymbol{T}}_{v},
\end{aligned}
$$

where $\Re($.$) is the real part operator.$

\section{ACKNOWLEDGMENT}

The authors would like to thank Philippe Réfrégier from Institut Fresnel for fruitful discussions and contributions. The authors would like to also thank the CNES (Centre National d'Études Spatiales) for funding the post-doctoral stay of Hélène Sportouche at Institut Fresnel in Marseille, France from 2012 to 2014.

\section{REFERENCES}

[1] T. Le Toan, S. Quegan, M. W. J. Davidson, H. Balzter, P. Paillou, K. P. Papathanassiou, S. Plummer, F. Rocca, S. Saatchi, H. Shugart, and L. Ulander, "The biomass mission: Mapping global forest biomass to better understand the terrestrial carbon cycle," Remote Sensing of Environment, vol. 115, pp. 2850-2860, nov 2011.

[2] S. R. Cloude and K. P. Papathanassiou, "Polarimetric SAR interferometry," IEEE Trans. Geosci. Remote Sensing, vol. 36, no. 5 , pp. 1151-1565, 1998.

[3] K. P. Papathanassiou and S. R. Cloude, "Single-baseline polarimetric SAR interferometry," IEEE Trans. Geosci. Remote Sens., vol. 39, pp. 2352-2363, Nov. 2001

[4] S. R. Cloude and K. P. Papathanassiou, "Three-stage inversion process for polarimetric SAR interferometry," IEE Proceedings, Radar, Sonar and Navigation, vol. 150, pp. 125-133, jun 2003.

[5] K. P. Papathanassiou and S. R. Cloude, "The effect of temporal decorrelation on the inversion of forest parameters from Pol-InSAR data," in IEEE International Geoscience and Remote Sensing Symposium (IGARSS), vol. 3, pp. 1429-1431, 2003.

[6] F. Garestier, P. Dubois-Fernandez, and I. Champion, "Forest Height Inversion Using High-Resolution P-Band Pol-InSAR Data," IEEE Trans. Geosci. Remote Sens., vol. 46, pp. 3544-3559, Nov. 2008.

[7] M. Lavalle and S. Hensley, "Extraction of structural and dynamic properties of forests from polarimetric-interferometric SAR data affected by temporal decorrelation," IEEE Trans. Geosci. Remote Sens., vol. 53, pp. 4752-4767, September 2015.

[8] R. N. Treuhaft and P. Siqueira, "Vertical structure of vegetated land surfaces from interferometric and polarimetric radar," Radio Science, vol. 35, pp. 141-177, jan-feb 2000.

[9] T. Flynn, M. Tabb, and R. Carande, "Direct estimation of vegetation parameters from Covariance data in polarimetric SAR interferometry," in Proc. IGARSS'02, pp. 1908-1910, IEEE, 2002

[10] M. Tabb, T. Flynn, and R. Carande, "Full maximum likelihood inversion of PolInSAR scattering models," in IEEE International Geoscience and Remote Sensing Symposium (IGARSS), vol. 2, pp. 1232-1235, IEEE, 2004.

[11] I. Hajnsek, F. Kugler, S.-K. Lee, and K. P. Papathanassiou, "Tropical-Forest-Parameter Estimation by Means of Pol-InSAR: The INDREX-II Campaign,” IEEE Trans. Geosci. Remote Sens. vol. 47, pp. 481-493, feb 2009

[12] S.-K. Lee, F. Kugler, I. Hajnsek, and K. P. Papathanassiou, "The impact of temporal decorrelation over forest terrain in polarimetric SAR interferometry," in International Workshop on Science and Applications of SAR Polarimetry and Polarimetric Interferometry (POLinSAR), 2009.

[13] F. Kugler, F. N. Koudogbo, K. Gutjahr, and K. P. Papathanassiou, "Frequency effects in Pol-InSAR forest height estimation," European Conference on Synthetic Aperture Radar (EUSAR), 2006.
[14] P. C. Dubois-Fernandez, T. Le Toan, S. Daniel, H. Oriot, J. Chave, L. Blanc, L. Villard, M. Davidson, and M. Petit, "The TropiSAR airborne campaign in French Guiana: Objectives, Description and Observed Temporal behavior of the Backscatter signal," IEEE Trans. Geosci. Remote Sens., pp. 1-14, 2012.

[15] G. Krieger, K. P. Papathanassiou, and S. R. Cloude, "Spaceborne polarimetric SAR interferometry: performance analysis and mission concepts," EURASIP J. Appl. Signal Process., vol. 2005, pp. 32723292, jan 2005.

[16] M. Lavalle, M. Simard, and S. Hensley, "A temporal decorrelation model for polarimetric radar interferometers," IEEE Trans. Geosci. Remote Sens., vol. 50, no. 7, pp. 2880-2888, 2012.

[17] H. A. Zebker and J. Villasenor, "Decorrelation in interferometric radar echoes," IEEE Trans. Geosci. Remote Sens., vol. 30, pp. 950 -959 , September 1992

[18] Y. Lei and P. Siqueira, "Estimation of forest height using spaceborne repeat-pass L-band InSAR correlation magnitude over the US State of Maine," Remote Sensing, vol. 6, no. 11, pp. 10252-10285, 2014.

[19] S. R. Cloude, "Robust parameter estimation using dual baseline polarimetric SAR interferometry," in IEEE International Geoscience and Remote Sensing Symposium (IGARSS), vol. 2, pp. 838-840, IEEE, 2002.

[20] S. R. Cloude and M. L. Williams, "A coherent EM scattering model for dual baseline POLInSAR," in IEEE International Geoscience and Remote Sensing Symposium (IGARSS), vol. 3, pp. 1423-1425, IEEE, 2003

[21] W. Shunjun, Z. Xiaoling, and H. Di, "Robust vegetation height extraction using maximum likelihood estimation for dual-baseline PolInSAR," in IEEE International Geoscience and Remote Sensing Symposium (IGARSS), vol. 2, pp. 633-636, IEEE, 2008.

[22] Y.-S. Zhou, W. Hong, F. Cao, Y.-P. Wang, and Y.-R. Wu, "Analysis of temporal decorrelation in dual-baseline POLinSAR vegetation parameter estimation," in IEEE International Geoscience and Remote Sensing Symposium (IGARSS), vol. 2, pp. 473-476, IEEE, 2008.

[23] S.-K. Lee, F. Kugler, K. P. Papathanassiou, and I. Hajnsek, "Quantification and compensation of temporal decorrelation effects in polarimetric SAR interferometry," in IEEE International Geoscience and Remote Sensing Symposium (IGARSS), pp. 3106-3109, IEEE, 2012.

[24] M. Lavalle and K. Khun, "Three-baseline InSAR estimation of forest height," IEEE Geosci. Remote Sens. Lett., vol. 11, no. 10, pp. 1737-1741, 2014.

[25] M. Neumann, L. Ferro-Famil, and A. Reigber, "Estimation of Forest Structure, Ground, and Canopy Layer Characteristics From Multibaseline Polarimetric Interferometric SAR Data," IEEE Transactions on Geoscience and Remote Sensing, vol. 48, pp. 1086-1104, Mar. 2010.

[26] S.-K. Lee, F. Kugler, K. P. Papathanassiou, and I. Hajnsek, "Quantification of temporal decorrelation effects at L-band for polarimetric SAR interferometry applications," IEEE Journal of Selected Topics in Applied Earth Observations and Remote Sensing, vol. 6, no. 3, pp. 1351-1367, 2013.

[27] R. N. Treuhaft, B. D. Chapman, J. R. Dos Santos, F. G. Gonçalves, L. V. Dutra, P. M. L. A. Graça, and J. B. Drake, "Vegetation profiles in tropical forests from multibaseline interferometric synthetic aperture radar, field, and lidar measurements," Journal of Geophysical Research-Atmospheres, vol. 114, dec 2009.

[28] R. N. Treuhaft, B. D. Chapman, J. R. Dos Santos, F. G. Gonçalves, L. V. Dutra, P. M. L. A. Graça, and J. B. Drake, "The ambiguity in forest profiles and extinction estimated from multibaseline interferometric SAR,” Boletim de Ciências Geodésicas, vol. 15, pp. 299312, jul-sep 2009

[29] A. Reigber and A. Moreira, "First demonstration of airborne SAR tomography using multibaseline L-band data," IEEE Trans. Geosci. Remote Sens., vol. 38, no. 5, Part 1, pp. 2142-2152, 2000.

[30] F. Lombardini and A. Reigber, "Adaptive spectral estimation for multibaseline SAR tomography with airborne L-band data," in IEEE International Geoscience and Remote Sensing Symposium (IGARSS), vol. 3, pp. 2014-2016, 2003.

[31] S. Tebaldini, "Algebraic synthesis of forest scenarios from multibaseline PolInSAR data," IEEE Trans. Geosci. Remote Sens., vol. 47, no. 12, pp. 4132-4142, 2009.

[32] S. Tebaldini, "Single and Multipolarimetric SAR Tomography of Forested Areas: A Parametric Approach," IEEE Trans. Geosci. Remote Sens., vol. 48, pp. 2375-2387, May 2010. 
[33] B. El Hajj Chehade and L. Ferro-Famil, "Polarimetric tomography for structural characterization of tropical forest," in European Radar Conference (EuRAD), pp. 180-183, IEEE, 2013.

[34] L. Ferro-Famil and S. Tebaldini, "ML tomography based on the MB RVoG model: Optimal estimation of a covariance matrix as a sum of two Kronecker products," in International Workshop on Science and Applications of SAR Polarimetry and Polarimetric Interferometry (POLinSAR), 2013

[35] A. Roueff, A. Arnaubec, P.-C. Dubois-Fernandez, and P. Réfrégier, "Cramer-Rao lower bound analysis of vegetation height estimation with random volume over ground model and polarimetric SAR interferometry," IEEE Geosci. Remote Sens. Lett., vol. 8, pp. 11151119, Nov. 2011.

[36] A. Arnaubec, A. Roueff, P.-C. Dubois-Fernandez, and P. Réfrégier "Influence of the nature of a priori knowledge on the precision of vegetation height estimation in polarimetric SAR interferometry," in EUSAR, Nuremberg, Germany, 2012.

[37] P. Réfrégier, A. Roueff, A. Arnaubec, and P.-C. Dubois-Fernandez, "Invariant Contrast Parameters of PolInSAR and Homogenous RVoG Model," IEEE Geosci. Remote Sens. Lett., vol. 11, pp. 14141417, Aug 2014.

[38] P. Garthwaite, I. Jolliffe, and B. Jones, Statistical Inference. London: Prentice Hall Europe, 1995.

[39] H. T. M. Dinh, T. Le Toan, F. Rocca, S. Tebaldini, M. M d'Alessandro, and L. Villard, "Relating P-band synthetic aperture radar tomography to tropical forest biomass," IEEE Trans. Geosci. Remote Sens., vol. 52, no. 2, pp. 967-979, 2014.

[40] S. Tebaldini, F. Rocca, M. M. d'Alessandro, and L. Ferro-Famil, "Phase Calibration of Airborne Tomographic SAR via Phase Center Double Localization," IEEE Trans. Geosci. Remote Sens., vol. 54 pp. 1775-1792, Mar 2016.

[41] S. Tebaldini and F. Rocca, "Multibaseline Polarimetric SAR Tomography of a Boreal Forest at P- and L-Bands," IEEE Transac tions on Geoscience and Remote Sensing, vol. 50, pp. 232-246, Jan. 2012.

[42] P. Stoica and R. Moses, Spectral Analysis of Signals. New Jersey: Prentice Hall, 2005

[43] R. A. Horn and C. R. Johnson, Topics in matrix analysis. Cambridge University Press, 1994.

[44] L. Ferro-Famil, M. Neumann, and Y. Huang, "Multi-baseline PolInSAR statistical techniques for the characterization of distributed media," in IEEE International Geoscience and Remote Sensing Symposium (IGARSS), vol. 3, pp. 971-974, 2009.

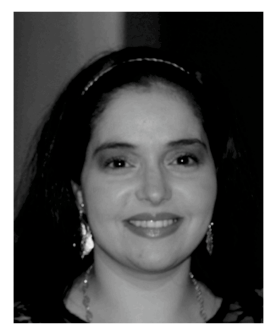

Hélène Sportouche received the Engineer degree in 2007 from Ecole Centrale Marseille, Marseille, France and the Ph.D. degree in signal and image processing in 2010 from Telecom ParisTech, Paris, France. She worked as R\&D engineer in optical and radar remote sensing imaging with Thales Communications (Massy, France) from 2007 to 2010 (Cifre agreement) then with Telecom ParisTech (Paris, France) from 2011 to 2012 (post-doctoral stay), and then with Centre National des Etudes Spatiales (Paris, France) and also with Institut Fresnel (Marseille, France) from 2012 to 2014 (post-doctoral stay). From 2016 to 2017, she worked with Altersis (Aix-en-Provence, France) and also with SuperSonic Imagine (Aix-en-Provence, France) in Ultrasound department as R\&D engineer (consultant position) in medical imaging. Since 2017, she is with SuperSonic Imagine (Aix-en-Provence, France) as Product Clinical Specialis in Product Management department, where she is participating in the definition as well as scientific and clinical development of innovating features and products.

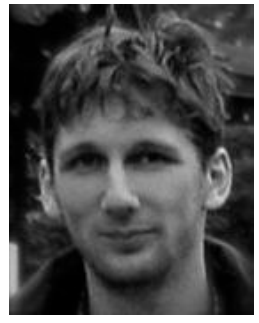

Antoine Roueff is presently associate profesor at Ecole Centrale de Marseille and member of the Fresnel Institute. He received his Ph.D. from the Institut National Polytechnique de Grenoble (France) in 2003 and worked as a post-doctoral researcher at the Commissariat à l'Energie Atomique (CEA) in Paris. His research activity concerns the development of statistical analysis of multi-component and multisensors signals. The application domains of his and geophysics. research are statistical optics, remote sensing

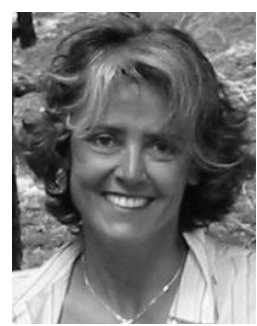

Pascale C. Dubois-Fernandez received the Diplôme d'Ingenieur from the Ecole Nationale Supérieure d'Ingénieur en Constructions Aéronautiques, Toulouse, France, in 1983 and the Master's and Engineering degrees from the California Institute of Technology, Pasadena, CA, USA, in 1984 and 1986, respectively. She joined the Radar Science and Technology Group with the Jet Propulsion Laboratory, Pasadena, CA, USA, where she stayed for ten years, participating in numerous programs such as Magellan, AIRSAR, and Shuttle Imaging Radar-C (SIR-C). She then moved back to France, where she worked on cartographic applications of satellite data. In 2000, she joined the Office National d'Etudes et de Recherches Aérospatiales (ONERA), Toulouse, France, as part of the Department of Electromagnetism and Radar, where she has been involved in the ONERA synthetic aperture radar (SAR) airborne platforms, RAMSES SETHI, and BUSARD, developing the science applications as the SAR Civilian Remote Sensing Expert. 